\title{
Results From Scotland's 2016 Report Card on Physical Activity for Children and Youth
}

\author{
John J. Reilly, Avril Johnstone, Geraldine McNeill, and Adrienne R. Hughes
}

\begin{abstract}
Background: The 2016 Active Healthy Kids Scotland Report Card aims to improve surveillance of physical activity (PA), facilitate international comparisons, and encourage evidence-informed PA and health policy. Methods: Active Healthy Kids Canada Report Card methodology was used: a search for data on child and adolescent PA and health published after the 2013 Scottish Report Card was carried out. Data sources were considered for grading if based on representative samples with prevalence estimates made using methods with low bias. Ten health behaviors/outcomes were graded on an $A$ to $F$ scale based on quintiles (prevalence meeting recommendations $\geq 80 \%$ graded $A$ down to $<20 \%$ graded $F$ ). Results: Three of the seven Health Behaviors and Outcomes received $F$ or $F$ - grades: Overall PA, Sedentary Behavior, and Obesity. Active and Outdoor Play and Organized Sport Participation could not be graded. Active Commuting to School was graded $C$, and Diet was graded $D$-. Family and Peer Influence was graded $D$-; Perceived Safety and Availability of Space for PA as well as the National Policy Environment were more favorable (both $B$ ). Conclusions: Grades were identical to those in 2013. Scotland has a generally favorable environment for PA, but children and adolescents have low PA and high sedentary behavior. Gaps in surveillance included lack of objectively measured PA, no surveillance of moderate-to-vigorous PA in children, summary surveillance data not expressed in ways which match recommendations (eg, for PA in young children; for screen-time), and no surveillance of Sport Participation, Active and Outdoor Play, or Sitting. Scottish policy does not include sedentary behavior at present.
\end{abstract}

Keywords: exercise, sedentary behavior, obesity, adolescent

A brief description of Scotland, and the rationale for an Active Healthy Kids Scotland Report Card, distinct from report cards produced by the other UK nations, was provided in the report on the 2013 Active Healthy Kids Scotland Report Card. ${ }^{1}$ Physical activity (PA) surveillance of children and adolescents in Scotland is based largely on the nationally representative Scottish Health Survey $\left(\mathrm{SHeS}^{2}\right)$, which uses self/parent-report measures of PA. For many years the $\mathrm{SHeS}$ has made the unlikely assumption that all reported PA is of moderate-to-vigorous intensity PA (MVPA), ${ }^{2,3}$ Annually collected SHeS data suggest that adherence to the 60 minutes/ day MVPA recommendation is very high in boys and girls during childhood, but falls dramatically in early adolescence, particularly in girls. National PA strategy, based explicitly on SHeS data, ${ }^{4,5}$ targets adolescents, particularly adolescent girls. However, the only validation study of SHeS methodology for measurement of PA in children found that MVPA was overestimated by 120 minutes/day on average, ${ }^{3}$ and the estimates of PA from the SHeS questionnaire were uncorrelated with accelerometer output in the same individuals. Moreover, recent longitudinal studies suggest that MVPA probably declines well before adolescence ${ }^{6,7}$. Current Scottish PA surveillance therefore provides a misleading basis for national policy, and there is need for a thorough critique of Scottish PA surveillance data, and Scottish policy, in this area.

Reilly, Johnstone, and Hughes are with the Physical Activity for Health Group, School of Psychological Sciences and Health, University of Strathclyde, Glasgow, Scotland. McNeill is Emeritus Professor of Public Health Nutrition, Institute of Applied Health Sciences, University of Aberdeen, Aberdeen, Scotland. Hughes (adrienne.hughes@strath.ac.uk) is corresponding author.
During 2013, the first Active Healthy Kids Scotland Report Card was developed and launched in both short-form ${ }^{8}$ and longform, ${ }^{9}$ as part of a Knowledge Translation project modeled closely on the Active Healthy Kids Canada Report Cards, ${ }^{10}$ organized by Active Healthy Kids Scotland (www.activehealthykidsscotland. co.uk). As noted in the 2013 Active Healthy Kids Scotland Report Card, ${ }^{1}$ child health in general, and PA in particular, has been a high priority in Scottish government policy. Scottish policy since 2013 has used the hosting of a major international sporting event, the 2014 Commonwealth Games, to promote PA among children and adolescents. ${ }^{4,5}$

The primary aim of the present paper is to summarize the process and results of the Active Healthy Kids Scotland 2016 Report Card. Secondary aims are to identify any changes in report card grades since 2013, to critique Scottish PA and health surveillance data for children and adolescents, and to critique any changes in PA and health surveillance and policy since 2013.

\section{Methods}

The 2016 Active Healthy Kids Scotland Report Card was produced by a RWG, based on the Canadian model ${ }^{10,11}$ which consisted of the 4 authors of the current article. The RWG was advised by a diverse group of stakeholders from many sectors (academia; health and education practice and policy; transport; sport; play), based on the approach taken in the Canadian card ${ }^{12}$ and in the 2013 Scottish card. ${ }^{1,8,9}$ Members of the Stakeholder Group commented on a draft version of the Scottish card. The 2016 Active Healthy Kids Scotland Report Card was cofunded by grants from 2 Scottish charities, The Robertson Trust (www.therobertsontrust.org.uk/) and Inspiring Scotland (www.inspiringscotland.org.uk). The funders 
have particular interests in using evidence to inform policy, and the promotion of child PA and health, but had no role in the content of the report card, and no role in the current manuscript.

The Active Healthy Kids Scotland Report Card in 2016 had the same 10 indicators (Table 1) as in 2013. Most of the indicators were health behaviors, but, as in 2013, the Research Working Group (RWG) felt that it was important to retain at least 1 health outcome (obesity) among the indicators, and 1 non-PA behavior (diet). Obesity has been a high priority of Scottish government policy for some time, and the inclusion of obesity provides an opportunity to increase the usefulness of the report card in Scotland. As in 2013, for some of the indicators there were multiple sources of Scottish surveillance data, and the aim was to base grades, where possible, on evidence which met the following criteria: data should be recent, published after the Active Healthy Kids Scotland Report Card in 2013; data should be derived, where possible, from nationally representative samples; data should be have minimal bias, obtained using methods which do not lead to large overestimates or underestimates of the prevalence of the health behavior or outcome.

During March and April 2016 the RWG searched for relevant evidence from Scotland, prioritizing nationally representative surveys as before. ${ }^{1}$ For the 10 indicators draft grades were assigned by the RWG during April 2016, by comparison of the national survey data against a relevant evidence-based recommendation (eg, 60 minutes MVPA/day, every day, for school-age children and adolescents) where this was available. The benchmark approach from the Active Healthy Kids Canada Report Cards ${ }^{10-12}$ was used: grade $A$ (we are succeeding with $\geq 80 \%$ of children and adolescents); grade $B$ (succeeding with 60 to $79 \%$ ); grade $C$ (succeeding with 40 to $59 \%$ ); grade $D$ (succeeding with 20 to $39 \%$ ); grade $F$ (succeeding with $<20 \%$ ); and INC (incomplete data). Each indicator was assigned a ' + ' if there was evidence that trends were improving since the last report card in 2013 or a '-' was assigned if there was evidence of worsening time trends since the last report card, and/or good evidence of marked socioeconomic inequalities for that indicator.

For each indicator we considered the probability of bias arising from error in the measures used. Where the probability and magnitude of bias were both high the measure was not used. For example, we declined to assign a grade to the SHeS surveillance measures of PA for the reasons given above, but graded this indicator based largely on a measure of MVPA from the Health Behavior in School Age Children (HBSC) 2014 Survey $^{13}$ which has a much smaller bias when used to assess adherence to PA recommendations. ${ }^{14,15}$ Decisions of this kind were made by the RWG, using a combination of their methodological expertise in the area, reference to recent reviews on biases in measurement of the various indicators, ${ }^{16-19}$ and the consultation process with stakeholders.

Draft report card grades were considered by the Stakeholder Group in May 2016. Stakeholders were asked to address the following questions, as before: ${ }^{9}$

Were any relevant Scottish data missed in the process of card development?

Were any data misinterpreted or misunderstood by the RWG (eg, were the draft grades justified)?

Were any relevant stakeholder groups or individuals omitted?

Which indicators not included in the card should be included in future cards?

The consultation process informed the final grades in the Active Healthy Kids Scotland 2016 Report Card, launched in June 2016.
Consultation comments, and our responses to them, are available on the project website (http://www.activehealthykidsscotland.co.uk).

\section{Results and Discussion}

The 2016 Active Healthy Kids Scotland Report Card grades are summarized in Table 1, and the 'cover story,' the card theme of Active and Outdoor Play, is summarized in Figure 1.The shortform report card, and a more detailed rationale for the indicators and grades, ${ }^{8,9}$ are both accessible from the project website (http:// www.activehealthykidsscotland.co.uk).

Five of the seven Health Behaviors and Outcomes Indicators (Table 1) could be graded with a combination of the availability of a

\section{Table 1 Grades According to Physical Activity Indicator in the 2016 Scottish Report Card on Physical Activity for Children and Youth*}

\begin{tabular}{lc}
\hline Indicator & Grades \\
\hline Overall Physical Activity Levels & $F$ \\
Organized Sport Participation & $I N C$ \\
Active and Outdoor Play & $I N C$ \\
Active Transportation & $C$ \\
Sedentary Behavior & $F$ \\
Family and Peer Influence & $D$ - \\
Obesity & $F-$ \\
Diet & $D-$ \\
Community and the Built Environment & $B$ \\
National Policies, Strategies and Investments & $B$
\end{tabular}

Note. The grade for each indicator is based on the percentage of children and youth meeting a defined benchmark: $A$ is $81 \%$ to $100 \%$; $B$ is $61 \%$ to $80 \%$; $C$ is $41 \%$ to $60 \%, D$ is $21 \%$ to $40 \% ; F$ is $0 \%$ to $20 \%$; INC is Incomplete data combined with lack of an evidence-based recommendation.

* All 2016 grades identical to 2013 grades.

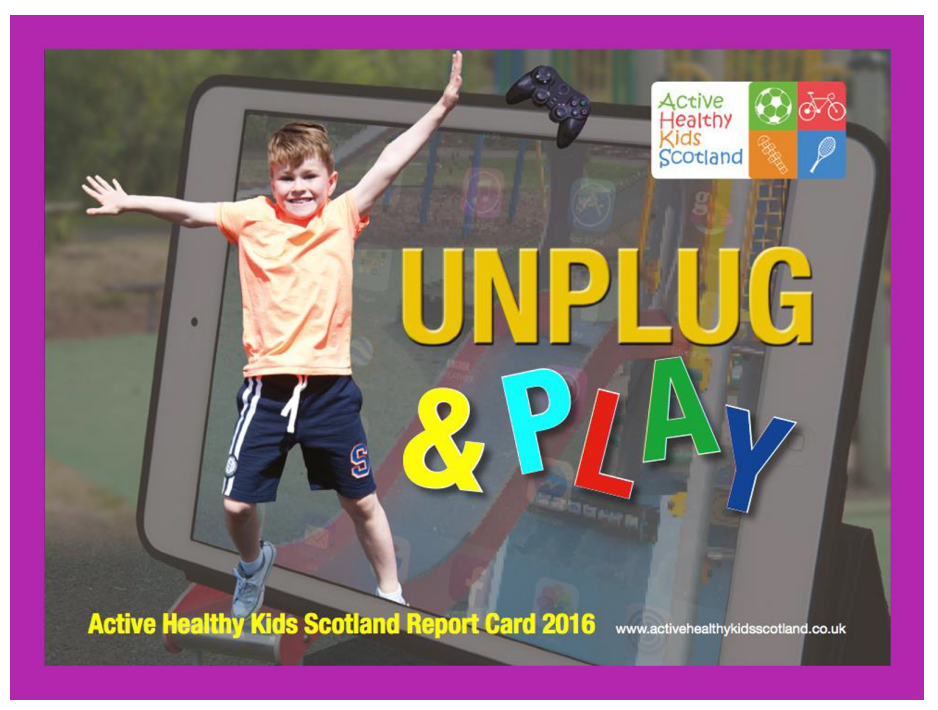

Figure 1 - Front cover of the 2016 Scottish Physical Activity Report Card. 
recommendation for that indicator, and a high degree of confidence in the benchmark of the percentage of children and adolescents meeting the guideline as noted above. The indicators Active and Outdoor Play and Organized Sport Participation could not be graded, in part because of a lack of an evidence-based recommendation for these behaviors, and in part because available Scottish data were limited or absent. ${ }^{9}$ Table 1 shows that the key health behaviors and outcomes were generally assigned low or 'fail' grades.

Grades for the indicators of Influences on Physical Activity and Health Behaviors and Outcomes were generally much better than grades for health behaviors and outcomes (Table 1). For the indicator Family and Peer Influence on Physical Activity Behaviors and Outcomes, no direct evidence of family or peer influence was available, and so proxy measures for the peer and adult health behaviors and outcomes had to be used, as in the 2013 report card. ${ }^{1}$ Scotland is characterized by obesity prevalence which is high, increasing, and socially patterned; adherence to adult PA recommendations is apparently moderate; adherence to dietary recommendations is low, socially patterned, and worsening over time. The indicator Community and the Built Environment (perceived safety, access to, and availability of space for PA) was graded $B$, reflecting the evidence that access and availability of space appeared to be generally favorable to PA, and perceived safety was moderately high. The indicator Policy referred to national policy only, and was graded $B$ on the grounds that Scotland has many national government policies, strategies, and investments which target most of the 7 health behaviors and outcomes included in the card (the notable exception in 2016 being sedentary behavior, as in 2013 ${ }^{1}$ ). Since the 2013 Scottish report card, national policy and strategy in this area has arguably improved, or at least increased, with greater recent emphasis on policy implementation including the signing of implementation agreements between national and local governments. ${ }^{5}$ In addition, recent Scottish policy in this area has sought to take advantage of the hosting of the 2014 Commonwealth Games to provide a population-wide PA 'legacy.'

\section{Key Health Behaviors and Outcomes Related to Physical Activity and Sedentary Behavior}

For children and adolescents reported exposure to recreational screen time was extremely high, well above the 2 hours per day recommended internationally. The 2 data sources on recreational screen time available for grading had reporting limitations: SHeS surveillance of recreational screen time expresses the prevalence of the child and adolescent population exceeding $4 \mathrm{~h} / \mathrm{d}$, not the $2 \mathrm{~h} / \mathrm{d}$ used in the screen-time recommendation ; ${ }^{2}$ the HBSC data are summarized for different forms of recreational screen time (TV viewing; gaming) separately, so estimating total recreational screen time for individuals is problematic. ${ }^{13}$ The overall PA grade was based on adolescents only, using MVPA data from the HBSC 2014. ${ }^{13}$ These data are based on a simple self-report method which has only a small bias for the assessment of adherence to MVPA recommendations, ${ }^{14,15}$ and suggest that around $30 \%$ of boys adhere to the recommendation at age 11 , and $15 \%$ at 15 ; in girls $21 \%$ adhered at age 11 and $11 \%$ at age $15 .{ }^{13}$ As noted above, surveillance of MVPA is not available for school-age children in Scotland as it is not measured in the SHeS. An additional problem identified by our critique of surveillance is that the SHeS erroneously uses school-age recommendations for PA when considering the adequacy of PA levels in preschool children. ${ }^{2}$

The low grades for the 'headline' report card indicators of PA and sedentary behavior, and in fact all grades in 2016, were the same as in 2013. ${ }^{1}$ The 2016 Active Healthy Kids Report Card Scotland therefore provides no evidence for an improvement in PA and health behaviors since the last report card, and there was some evidence of a possible worsening of some of the indicators, discussed briefly below.

The 'cover story' for the 2016 Active Healthy Kids Scotland Report Card ${ }^{8,9}$ (Figure 1) was Active and Outdoor Play. The rationale for the cover story was that active and outdoor play represents a potentially important opportunity to increase MVPA. ${ }^{20}$ Active and outdoor play is a neglected domain of PA in PA promotionresearch and policy efforts have focused on school-based domains (notably PE, a high policy priority in Scotland) ${ }^{4,5}$ and no Scottish data sources exist for active and outdoor play specifically. An additional argument for surveillance of active and outdoor play, and consideration of this domain in PA policy, is that differences in MVPA between children in high-income versus low-income countries might be partially attributable to differences in the amount of time spent in active and outdoor play. ${ }^{21,22}$ In addition, recent systematic reviews suggest that the school-based domains (PE, recess, active commuting to school) which have been the focus of most previous research and policy effort may contribute relatively little to population MVPA at present. ${ }^{23-25}$ Comparisons of the likely effect of different interventions on population MVPA ${ }^{20,26}$ also suggest that future efforts should extend beyond the school.

Scottish government surveillance and policy is based heavily on the SHeS. ${ }^{4,5}$ The critique of SHeS data and data interpretation for the current study has highlighted multiple weaknesses in the SHeS data collection, data presentation, and data interpretation. For example, Organized Sport Participation is not measured specifically by the $\mathrm{SHeS}$, despite the prominence of the potential Commonwealth Games legacy in policy. As noted above, the SHeS does not measure Active and Outdoor Play specifically. A serious weakness of the SHeS data interpretation is that the survey attempts to measure total volume of $\mathrm{PA}^{2}$ but data on total volume of PA are erroneously treated as time spent in MVPA when used in surveillance and in evidence-based policy. ${ }^{4,5}$ As a result, apparent adherence to MVPA recommendations based on this misinterpretation of SHeS data are extremely high: $80 \%$ of boys and $73 \%$ of boys at age 11 to 12 , with even higher apparent adherence in children according to the SHeS. 2,4,5 These estimates greatly exceed those for the same age from the HBSC Surveys, ${ }^{13}$ as well as those from the other countries in the UK, ${ }^{27-29}$ and suggest that typical levels of MVPA among Scottish children are similar to those of children from rural Mozambique. ${ }^{30}$ No objectively measured Scottish MVPA data were available across the child and adolescent age range. Finally, SHeS data on preschool children are compared against the MVPA recommendations for school-age children and adolescents in the $\mathrm{SHeS}^{2}$ this is inappropriate, and the lack of distinction between preschool and school-age children is also inconsistent with the increasing emphasis on the early years in Scottish government policy.

Multiple sources of Scottish data were available, as before, ${ }^{1}$ on active commuting (walking, cycling) to and from school, and these data sources were highly consistent in suggesting that that around $50 \%$ of Scottish primary school children normally commute actively to school, and $40 \%$ to $45 \%$ of those at high school commute actively, hence a $C$ grade was assigned. New Scottish data on active commuting to nursery became available since the 2013 report card, and this suggested that around half of preschool children commute actively. ${ }^{8,9}$ Surveillance effort on active commuting to school/ nursery in Scotland is substantial, based on multiple surveys, and 
some of the surveys are likely to be redundant. A gap identified by the current study is that active commuting to other locations, on the 160 or so days per year that children are not at school, is not included in current surveillance.

\section{Key Influences on Health Behaviors and Outcomes}

The $D$ - grade for the indicator Family and Peer Influence on Physical Activity Behaviors and Outcomes was limited to proxy data because we were unable to find direct Scottish evidence of parental or peer influence based on measures of PA or diet with minimal bias. The proxy data indicates that Scottish children and adolescents develop among adult norms of overweight and obesity, obesogenic diet, but apparently moderately high levels of PA. Peer norms of low PA and high exposure to screen time suggest that peer influences are also unfavorable.

The $B$ grade for the indicator Community and the Built Environment was based on evidence ${ }^{8,9}$ that Scottish children and adolescents have high perceived access to/availability of space for PA, and perceived safety of such space was moderately high. ${ }^{1,8,9}$ The national policy environment was graded $B$. Many Scottish policies are relatively recent, and they may need greater time and/or greater implementation efforts if they are to impact on the generally unfavorable grades for the indicators in the Health Behaviors and Outcomes category. The emphasis on implementation of policy in Scotland has increased since the last report card, with the recent signing of implementation agreements between national and local government. ${ }^{5}$ The major gaps in the national policy environment were the absence of any policies on sedentary behavior, and the lack of emphasis on evaluation of policy implementation.

\section{Obesity and Diet Indicators}

Obesity prevalence (graded $F$-), as defined by BMI percentile, is much higher than in the past, ${ }^{1,8,9}$ is more prevalent among the more socioeconomically deprived, and socioeconomic inequality in child and adolescent obesity appears to be widening. In addition, systematic reviews of obesity diagnostic studies have shown that obesity prevalence estimates using BMI are highly conservative. ${ }^{18,19}$ Since the 2013 Active Healthy Kids Report Card Scotland was published new Scottish data (from the UK Diet and Nutritional Surveys 2008-2012 $2^{31}$ ) have become available on prevalence of overweight and obesity among 2- to 3-year-olds: these estimates are based on the WHO BMI standards ${ }^{32}$ and suggest very high prevalence (17\% prevalence of obesity at age 2 to 3 years).

The 2014 Scottish Health Survey ${ }^{2}$ showed little change in children's fruit and vegetable consumption which remained at an average of 2.8 portions per day. Data for 2012/2013/2014 combined showed that around half the children had nondiet soft drinks once a day or more while over a third had potato chips once a day or more, with the highest proportions among children in the most socioeconomically deprived areas. Among adults there was no evidence for improvement in diet over the period 2008 to 2014 and some evidence of widening socioeconomic disparities. Data for children from 2003 to 2012/2013, however, suggest some gradual improvements in children's diets (eg, with an increase in the frequency of oily fish consumption and decrease in frequency of consumption of potato chips). The UK-wide National Diet and Nutrition Survey has recently released data for children in Scotland for the period 2008-2012 which show higher than recommended intakes of saturated fat and particularly of nonmilk extrinsic sugar (broadly similar to added sugar) which was on average $15.8 \%$ of energy intake in boys and $14.9 \%$ of energy intake in girls (ie, the average is 3 times higher than the recently revised recommended figure of $5 \%$ of food energy). As part of the critique of methods for the current study we note that, while all dietary data methodology is limited, nutritionists would generally have greater confidence in the methods used in the National Diet and Nutrition Survey (prospective diet diaries) than in the methods used in the SHeS (food-frequency questionnaires).

\section{Strengths and Limitations}

Grades assigned in the 2016 Active Healthy Kids Scotland Report Card were based on an expert critique of the best (ie, most recent, most representative, least biased) available data using the robust methodology developed for the Active Healthy Kids Canada Report Cards. ${ }^{11,12}$ For a number of indicators grading was either problematic or impossible: high quality evidence was lacking in some cases (eg, MVPA of primary school age children not available), so the grade was based largely on adolescent data, as in 2013; no evidence was available for some indicators (eg, there was no evidence on Organized Sport Participation ${ }^{8,9}$ ); indirect evidence had to be used for grading in other cases (eg, for Family and Peer Influence as noted above; for Active and Outdoor Play no specific surveillance exists and no clear evidence-based recommendations exist for the behavior). It is hoped that the identification and highlighting of these gaps and weaknesses in the evidence will lead to improved PA and health surveillance in Scotland in future.

\section{Conclusions}

The 2016 Active Healthy Kids Scotland Report Card provides further evidence for the high-income nation paradox identified by the comparison of Active Healthy Kids Report Cards from 15 nations in 2014. ${ }^{21}$ Despite some limitations in the evidence it is clear that Scotland has a generally favorable built and policy environment for child and adolescent PA, but low child and adolescent PA. In contrast, low-income nations generally have very unfavorable environments, but higher levels of child and adolescent PA. Lessons from such international comparisons should be helpful in Scottish policy in this area in future.

\section{Acknowledgments}

The authors thank members of the Stakeholder Group for their varied and substantial contributions to the card. This work was supported by Knowledge Exchange Grants from Inspiring Scotland and The Robertson Trust.

\section{References}

1. Reilly JJ, Dick S, McNeill G, Tremblay MS. Results from the Scottish Report Card on Physical Activity for Children and Youth. J Phys Act Health. 2014;11(Suppl 1):s93-s97. PubMed doi:10.1123/jpah.20140183

2. Scottish Health Survey 2014. www.scotland.gov.uk/Topics/Statistics/ Browse/Health/scottish-health-survey. Accessed March 31, 2016.

3. Basterfield L, Adamson AJ, Parkinson KN, Maute U, Li PX, Reilly JJ. Surveillance of physical activity in the UK is flawed: validation of the Health Survey for England physical activity questionnaire. Arch Dis Child. 2008;93:1054-1058. PubMed doi:10.1136/adc.2007.135905

4. Active Scotland Outcomes Framework. www.gov.scot/Topics/ArtsCultureSport/Sport/Outcomes-Framework. Accessed June 1, 2016. 
5. Murray A, Calderwood C, O' Connor N, Mutrie C. Scotland's progress in putting policy about physical activity into practice. Br J Sports Med. 2016;50(6):320-1. doi:10.1136/bjsports-2015-095744 PubMed

6 . Reilly JJ. When does it all go wrong?: longitudinal studies of changes in moderate-to-vigorous intensity physical activity across childhood and adolescence. J Exerc Sci Fit. 2016;14(1):1-6.

7. Basterfield L, Pearce MS, Parkinson KN, Adamson AJ, Reilly JJ. Longitudinal study of physical activity and sedentary behavior in children. Pediatrics. 2011;127:e24-e30. PubMed doi:10.1542/peds.2010-1935

8. The 2013 and 2016 Active Healthy Kids Scotland Report Cards (Short Form). Glasgow: Active Healthy Kids Scotland. www.activehealthykidsscotland.co.uk. Accessed March 31, 2016.

9. The 2013 and 2016 Active Healthy Kids Scotland Report Cards (Long Form). Glasgow: Active Healthy Kids Scotland. www.activehealthykidsscotland.co.uk. Accessed March 31, 2016.

10. Colley RC, Brownrigg M, Tremblay MS. A model of knowledge translation in health: The Active Healthy Kids Canada Report Card on Physical Activity for Children and Youth. Health Promot Pract. 2012;13:320-330. PubMed doi:10.1177/1524839911432929

11. Barnes JD, Colley RC, Tremblay MS. Results from the Active Healthy Kids Canada 2011 Report Card on Physical Activity for Children and Youth. Appl Physiol Nutr Metab. 2012;37:793-797. PubMed doi:10.1139/h2012-033

12. Active Healthy Kids Canada. Are We Driving Our Kids to Unhealthy Habits? The 2013 Active Healthy Kids Canada Report Card on Physical Activity for Children and Youth. Toronto: Active Healthy Kids Canada; 2013.

13. Health Behaviour in School-Aged Children Scotland National Report 2014 (HBSC 2014).

14. Hardie Murphy M, Rowe DA, Belton S, Woods CB. Validity of a 2-item questionnaire for assessing attainment of physical activity guidelines in youth. BMC Public Health. 2015;15:1080. PubMed doi:10.1186/ s12889-015-2418-6

15. Ridgers N, Timperio A, Crawford D, Salmon J. Validity of a brief self-report instrument for assessing compliance with physical activity guidelines amongst adolescents. J Sci Med Sport. 2012;15:136-141. PubMed doi:10.1016/j.jsams.2011.09.003

16. Lubans DR, Hesketh K, Cliff DP, et al. A systematic review of the validity and reliability of sedentary behaviour measures used with children and adolescents. Obes Rev. 2011;12:781-799. PubMed doi:10.1111/j.1467-789X.2011.00896.x

17. Reilly JJ, Kelly J, Wilson DC. Accuracy of simple clinical and epidemiological definitions of childhood obesity: systematic review and evidence appraisal. Obes Rev. 2010;11:645-655. PubMed doi:10.1111/j.1467-789X.2009.00709.x
18. Reilly JJ, Penpraze V, Hislop J, Davies G, Grant S, Paton JY. Objective measurement of physical activity and sedentary behaviour: review with new data. Arch Dis Child. 2008;93:614-619. PubMed doi:10.1136/ adc. 2007.133272

19. Javed A, Jumean MH, Murad D, et al. Diagnostic performance of BMI to identify obesity as defined by adiposity in children and adolescents: systematic review and meta-analysis. Pediatr Obes. 2015;10:234-244. PubMed doi:10.1111/ijpo.242

20. Janssen I. Active play: an important physical activity strategy in the fight against childhood obesity. Can J Public Health. 2014;105:e22e27. PubMed doi:10.17269/cjph.105.4154

21. Tremblay MS, Gray CE, Akinroye K, et al. Physical activity of children: a global matrix of grades comparing 15 countries. J Phys Act Health. 2014;11(suppl 1):s113-s125. PubMed doi:10.1123/jpah.20140177

22. Katzmarzyk PT. Increasing global research capacity in physical activity for children and youth. Res Exerc Epidemiol. 2014;16:71-75.

23. Hollis JL, Williams AJ, Sutherland R, et al. A systematic review and meta-analysis of MVPA levels in elementary school PE lessons. Prev Med. 2016;86:34-54.

24. Reilly JJ, Johnston G, McIntosh S, Martin A. Contribution of school recess to daily physical activity: systematic review and evidence appraisal. Health Behav Policy Rev. 2016;3:581-589.

25. Martin A, Boyle J, Corlett F, Kelly P, Reilly JJ. Contribution of walking to school to individual and population moderate-vigorous intensity physical activity: systematic review and meta-analysis. Pediatr Exerc Sci. 2016;28(3):353-363. PubMed doi:10.1123/pes.2015-0207

26. Bassett DR, Fitzhugh EC, Heath GW, et al. Energy expenditure for school-based policies and active living. Am J Prev Med. 2013;44:108113. PubMed doi:10.1016/j.amepre.2012.10.017

27. WHO Europe 2015. United Kingdom of Great Britain and Northern Ireland Physical Activity Factsheet.

28. British Heart Foundation. Physical Activity Statistics 2015. www.bhf. org.uk. Accessed March 31, 2016.

29. UK Association for Physical Education. Health Position Paper 2015.

30. Prista A, Picardo S, Ribiero E, et al. Results from Mozambique's 2014 Report Card on Physical Activity for Children and Youth. J Phys Act Health. 2014;11(suppl 1):s79-s82. PubMed doi:10.1123/jpah.20140179

31. Food Standards Agency. National Diet \& Nutrition Survey Rolling Programme Results from Years 1-4 combined for Scotland 2008/92011/12. www.food.gov.uk. Accessed March 31, 2016.

32. WHO Multicentre Growth Reference Study Group, and de Onis M. WHO Child Growth Standards based on length/height, weight, and age. Acta Paediatr. 2006;95(suppl 450):76-85. 